\section{AB1031 ETANERCEPT-INDUCED LUPUS-LIKE SYNDROME IN THE BOY WITH IDIOPATHIC DERMATOMYOSITIS OR DELAYED MANIFESTATION OF SYSTEMIC LUPUS ERYTHEMATOSUS?}

Maria Kaleda, Irina Nikishina. V. A. Nasonova Research Institute of Rheumatology, Pediatric, Moscow, Russian Federation

Background: Treatment of juvenile onset rheumatic diseases (RD) is an ongoing problem because of the more aggressive disease course and more various clinical manifestations than adult-onset RD. Since the main clinical symptoms may overlap between well-known various RD, in some cases the path to diagnosis and the search for optimal therapy takes longer, especially in patients of pre-adolescence and adolescence.

Objectives: We described a rare case of one boy with RD who presented initially with severe dermatomyositis with sequential evolution to systemic lupus erythematosus.

Methods: Case report.

Results: A 8 yo male patient with normal physical, psychosocial and cognitive development experienced muscle weakness, periorbital erythema, Gottron symptom, dysphagia in September 2015 y, which developed after insolation. When he was admitted to hospital, increased levels of musclederived enzymes and positivity of ANA 1:640 were found. He was diagnosed as having juvenile dermatomyositis and was treated with prednisolone $(\mathrm{Pr})$ for 3 years (IV courses repeated and per os constantly, max dose $1.5 \mathrm{mg} / \mathrm{kg} /$ day, min dose $0.5 \mathrm{mg} / \mathrm{g} /$ day), IVIG courses repeated and sequentially methotrexate, hydroxychloroquine, cyclosporine with short time of remission. Muscle weakness disappeared after the treatment, but in October 2015 y he experienced new eruptions on his face, heavy aphtosis stomatitis, lymphadenopathy, distal polyneuropathy, weight loss without strong effect after increase the dose of $\mathrm{Pr}$, IVIG courses repeated, cyclosporine and added of abatacept during 6 months. Because the response to this treatment was low, etanercept was started in June $2016 \mathrm{y}$, but after two injections he had flare of disease: bright rash on the face, scalp and legs, hard aphthosis stomatitis, cheilitis, cognitive disorders, diffuse alopecia. He received IV course of $\operatorname{Pr}$ without significant stable effect. We reviewed diagnosis as systemic lupus erythematosus and changed therapy in November of 2016. Because he had steroid's osteoporosis with spondylopathy, this combination that made us initiate treatment with mycophenolate mofetil and repeating courses of rituximab (3 courses in total) without increased dose of Pr. Clinical features gradually improved and now he feel goog and receive mycophenolate mofetil and Pr $5 \mathrm{mg} /$ day.

Conclusion: This clinical observation can be considered as a course of overlap-syndrome with a successive phase change that could be triggered by anti-TNF therapy, or as an unusual course of SLE in a male patient with a predominance of myositis at onset. Treatment strategy, including of $\mathrm{Pr}$, mycophenolate mofetil and rituximab in a young patient proved successful for treatment and seems to be justified in similar cases.

Disclosure of Interests: None declared

DOI: 10.1136/annrheumdis-2019-eular.4752

\section{AB1032 1 FACTORS TO AID IN EARLY DIAGNOSIS OF KIKUCHI'S DISEASE IN ASIAN CHILDREN}

Poh Lin Pauline Chan Ng, Lee Kean Lim, Elizabeth Ang, Pei Ling Ooi. National University Hospital, Khoo Teck Puat-National University Children's Medical Institute, Singapore, Singapore

Background: The diagnosis of Kikuchi's Disease (KD), also known as histiocytic necrotizing lymphadenitis, is often delayed as patients often undergo multiple investigations to exclude other causes of cervical lymphadenopathy, such as infection and malignancy ${ }^{1}$

Objectives: To describe the clinical features and treatment response of pediatric patients diagnosed with KD in our institution to aid early recognition.

Methods: A retrospective study was conducted on patients < 18 years old diagnosed with KD in National University Hospital, Singapore, from Jan 2006 - Dec 2018. Demographics, clinical characteristics and treatment information were collected.

Results: 14 patients were diagnosed with KD. All were Asian and majority were Chinese (64.2\%). $57.1 \%$ were male and the age of presentation ranged between 2.8 to 17.9 years old. Fever duration at diagnosis ranged between 8 - 50 days. Median height of temperature was 39.8 (IQR: 39.4 - 40.0) deg C. This was frequently associated with neck pain and swelling $(57.1 \%)$ and constitutional symptoms $(71.4 \%)$ such as anorexia and weight loss. Other symptoms include abdominal symptoms of pain, vomiting or diarrhea $(57.1 \%)$, rash $(35.7 \%)$ and oral ulcers $(28.6 \%)$.
All patients had cervical lymphadenopathy either in the anterior cervical triangle or both anterior and posterior triangles, but not in the posterior triangle alone. This was usually multiple $(92.9 \%)$, measuring $\geq 2 \mathrm{~cm}$ in diameter $(100 \%)$, tender $(85.7 \%)$, and tended to be unilateral $(64.3 \%)$; none had overlying warmth or skin changes. $14.3 \%$ had isolated hepatomegaly while $14.3 \%$ had hepatosplenomegaly.

Common lab abnormalities included cytopaenias (50\% bicytopaenia, $28.6 \%$ leucopaenia, $7.1 \%$ anaemia), elevated inflammatory markers $\mathrm{C}$-reactive protein (CRP) (range: $12-117 \mathrm{mg} / \mathrm{L}$ ), erythrocyte sedimentation rate (ESR) (range $15-116 \mathrm{~mm} / \mathrm{h}$ ) and lactate dehydrogenase (range: 480$1514 \mathrm{U} / \mathrm{L})$. Only $3 / 14$ had mild transaminitis. Interestingly, almost half had low alkaline phosphatase (ALP). All patients had normal to high complements and were negative for anti-dsDNA antibodies. Ultrasound of the neck typically revealed non-specific findings of reactive lymphadenopathy while 1 showed suspicious features of thickened cortex and loss of fatty hilum. 10/14 patients underwent excision biopsy which confirmed the diagnosis of Kikuchi Disease on histology. 3/14 did not have excision biopsy as they responded promptly to non-steroidal anti-inflammatory drugs (NSAIDs), one declined excision biopsy.

All patients had received empiric antibiotics with no response. $64.3 \%$ required treatment with NSAIDs for an average of 2-4 weeks. Of the 4 patients who required steroid therapy, one was started at the diagnosis of $K D$, while the other 3 received steroids upon failure of NSAIDs Response to either NSAIDs or prednisolone, if effective, was usually prompt, within 24 hours.

Conclusion: Prolonged high grade fever with multiple tender cervical lymphadenopathy $\geq 2 \mathrm{~cm}$ without warmth or redness and non-response to antibiotics, should raise concerns for KD. The presence of cytopaenia with moderately elevated inflammatory markers and LDH, coupled with low ALP, may be considered supporting biochemical features of KD. Prompt response to a trial of NSAIDs can help with the diagnosis of KD and potentially avoid excision biopsy of the lymph node.

More research needs to be done to determine a clinical and biochemical diagnostic criteria for Kikuchi's Disease so as to aid early diagnosis and potentially avoid invasive excision biopsy of the lymph nodes.

\section{REFERENCES}

[1] Lin HC, et al. Kikuchi's disease in Asian children. Pediatrics. 2005 Jan;115 (1):e92-6.

[2] Chen CK, et al. Kikuchi disease in Asian children. J Paediatr Child Health. 2006 Mar;42(3):104-7.

Disclosure of Interests: None declared

DOI: 10.1136/annrheumdis-2019-eular.7641

\section{AB1033 MEASUREMENT OF DISEASE ACTIVITY LEVEL OVER TIME IN CHILDREN WITH JUVENILE IDIOPATHIC ARTHRITIS UNDER BIOLOGIC TREATMENT, USING THE JUVENILE DISEASE ACTIVITY SCORE HUNGARIAN DATA FROM THE JUVENILE IDIOPATHIC ARTHRITIS REGISTRY OF THE NATIONAL INSTITUTE OF RHEUMATOLOGY AND PHYSIOTHERAPY}

llonka Orbán ${ }^{1}$, Krisztina Sevcic ${ }^{1}$, Emese Virág Kiss ${ }^{1}$, Gyula Poór ${ }^{2} .{ }^{1}$ National Institute of Rheumatology and Physiotherapy, Department of Clinical Immunology, Adult and Paediatric Rheumatology, Budapest, Hungary, ${ }^{2}$ National Institute of Rheumatology and Physiotherapy, 1st Department of Rheumatology, Budapest, Hungary

Background: A wide variety of measures are currently available to monitor the disease course over time in children with JIA.The Juvenile Disease Activity Score JADAS71 version was validated for 71 joints, global score for the JADAS71 is: $0-101$.

Objectives: To report the changes of disease activity in JIA patients under biological treatment at age 18, using the JADAS 71 score for the extended oligoarticular and seropositive/seronegative polyarticular form of JIA.

Methods: The Hungarian Biologics JIA Registry of National Institute of Rheumatology and Physiotherapy, Budapest is a longitudinal, unicenter, observational study, that has been maintained since 2002. JIA-ILAR criteria were used for the diagnosis.Patients with polyarticular course, who failed to respond or did not tolerate methotrexate and were treated with biological agents at the Paediatric Rheumatologic Centre of the National Institute of Rheumatology and Physiotherapy, Budapest, Hungary from 2002 to 25th January,019, were enrolled in an open label observational study and have been monitored prospectively in the registry. At baseline patients and disease characteristics were registered such as, patients 